\title{
Virtual collaborative design environment: supporting seamless integration of multitouch table and immersive VR
}

Article

Accepted Version

Roupé, M., Johansson, M., Maftei, L., Lundstedt, R. and Viklund-Tallgren, M. (2020) Virtual collaborative design environment: supporting seamless integration of multitouch table and immersive VR. Journal of Construction Management and Engineering, 146 (12). ISSN 1943-7862 doi: https://doi.org/10.1061/(ASCE)CO.1943-7862.0001935 Available at https://centaur.reading.ac.uk/95619/

It is advisable to refer to the publisher's version if you intend to cite from the work. See Guidance on citing.

To link to this article DOI: http://dx.doi.org/10.1061/(ASCE)CO.19437862.0001935

Publisher: American Society of Civil Engineers

All outputs in CentAUR are protected by Intellectual Property Rights law, including copyright law. Copyright and IPR is retained by the creators or other copyright holders. Terms and conditions for use of this material are defined in the End User Agreement. 


\section{CentAUR}

Central Archive at the University of Reading

Reading's research outputs online 
1 Virtual Collaborative Design Environment (ViCoDE): supporting seamless

2 integration of multi-touch table and immersive VR

Mattias Roupé, roupe@chalmers.se, Chalmers University of Technology, SE-412 96 Gothenburg, Sweden Mikael Johansson, jomi@chalmers.se, Chalmers University of Technology, SE-412 96 Gothenburg, Sweden Laura Maftei, , 1.maftei@ reading.ac.uk, University of Reading, Whiteknights., RG6 6DF, Reading, UK Rikard Lundstedt, rikard.lundstedt@design.lth.se, Lund University, Box 117, SE-221 00, Lund, Sweden Mikael Viklund-Tallgren, mikael.tallgren@chalmers.se, Chalmers University of Technology, SE-412 96, Gothenburg, Sweden

Abstract. During the design of a new hospital it is necessary for all involved stakeholders to understand, participate, communicate, and collaborate with each other to obtain a high-quality outcome. In order to support these creative and shared design processes, this paper presents the design and evaluation of Virtual Collaborative Design Environment (ViCoDE) - a new collaborative design system. ViCoDE features seamless integration of a multi-touch table and several immersive VR-systems that facilitates interactive and collaborative design work with immediate feedback. The system has been evaluated during two collaborative design workshops in a real-life context of designing new healthcare environments. The results show that the multi-touch table and VR-system complement each other very well by facilitating different design spaces both collaborative, as well as individual - and that it fosters better understanding, participation, communication, knowledge sharing and collaboration among the different stakeholders. The contribution is two folded: presentation of new a collaborative design system and the evaluation of the use of it in a real-life design context, which demonstrate how these new technology-based workshops may facilitate design management.

Keywords: Virtual Reality, Collaborative Design, Collaborative Virtual Environments, CSCW.

\section{Introduction}

When designing new healthcare environments and hospitals, many different stakeholders and specialists from healthcare and construction are involved with different experiences, knowledge levels and ability to interpret information. In these design processes there are also pressures to reduce lead-time and costs, and to minimize defects and design errors, to finally increase client satisfaction and quality of the new facility (Elf et al. 2015; Lindahl and Ryd 2007). Furthermore, it has been recognized that construction projects are becoming more 
difficult to manage due to their increasing complexity and technologically advanced buildings (Bryde et al. 2013; Chien et al. 2014; Winch 2010). Consequently, it is important to achieve better communication and understanding between disciplines (Bhatla and Leite 2012; Eastman 2016). There is also an increase in complexity in today's design and its amount of information (Van Berlo and Natrop 2015). The most common information media in these processes are documents, descriptions, 2D-drawings and pictures. However, these media can be difficult to interpret and understand, placing high cognitive demands on the stakeholders/viewer's ability to transform the information into a self-made mental image of the project (Roupé 2013). The self-made mental image could also be misinterpreted and it may differ depending on the individual's background, education, experience and interest (Roupé 2013). This means that important feedback from healthcare specialists (e.g. surgeons, nurses) can be lost during the planning and design process. These malfunctions are detected too late in the process, when the healthcare environment is already built (Elf et al. 2015; Lindahl and Ryd 2007).

One potential solution to this problem is to take advantage of immersive Virtual Reality (VR) instead of traditional 2D-drawings and pictures. Although the use of VR-technology has been limited in the past due to lack of available 3D data from the design process, the recent introduction of Building Information Models (BIM) within the AEC field has opened up new possibilities, by enabling extraction of 3D data directly from the architect's own design environment (Van Berlo and Natrop 2015; Du et al. 2018a; b; Eastman 2016; Johansson et al. 2014, 2015; Liu et al. 2020; Mastrolembo Ventura et al. 2019; Mastrolembo Ventura and Castronovo 2018; Roupé et al. 2016; Xue et al. 2012). In this context, Du et. al. (2018a;b) focused on mapping objects in VR to the original objects in BIM authoring tools so the users can easily synchronize changes in from authoring tool (e.g. Autodesk Revit) to a visualization environment. Furthermore, use of real-time visualizations has become more accessible in practice (Johansson et al. 2014, 2015). With the use of a Head-Mounted Display (HMD) the different stakeholders can move around and experience the future planned healthcare environment in scale 1:1 and therefore share a common frame of reference (Paes et al. 2017; Roupé et al. 2016). However, with HMDs being primarily a tool for the individual, it makes it less suitable for active collaborative design work, which also relies much on face-to-face communication and gestures (Gugenheimer et al. 2017; Wang and Dunston 2008). In this context, it is also important to allow participants to express ideas and thoughts to the other members of the team by performing actual changes to the design.

In order to address the current situation this paper presents a new collaborative design system which uses a seamlessly connected multi-touch table and several VR-systems for interactive and collaborative design. In addition to describing technical details of the system, we present and discuss the results from using the system 
during two design workshops held as part of an ongoing design of two new hospitals. By assessing both technical and social factors around the use of this technology in a realcontext, these results contribute to the body of knowledge on how and these new types of collaborative design systems and collaborative design workshops facilitate the design management process.

\subsection{Literature Review}

The rising complexity of construction projects sets new demands on how design should be conducted. Computer-Supported Cooperative Work (CSCW) approaches are often based on the assumption that complex problems require more knowledge than any single individual possesses and in this context it is necessary for all involved stakeholders to participate, understand, communicate and collaborate with each other to obtain a higher quality outcome (Arias et al. 2000; Fisher et al. 2005).

These types of design problems are often moving targets that do not have straightforward solutions but only have resolutions during the design process, and the context in which these problems exist is characterized by change, conflict, and multiple stakeholders (Arias et al. 2000; Heldal and Roupé 2012).The resolution of design problems grows out of the shared understanding that emerges as different stakeholders begin to better understand each other's perspectives (Arias et al. 2000; Fisher et al. 2005). Still, communication breakdowns are often experienced because stakeholders have different interests and agendas and belong to different cultures that use different norms, symbols, and representations. However, by creating a shared understanding through collaborative design, it is possible to provide opportunities and resources for design activities embedded in a social creative design process in which all actors can actively contribute rather than having passive consumer roles (Arias et al. 2000; Fisher et al. 2005).

As a way to facilitate and improve collaboration across different stakeholders, Collaborative Virtual Environments (CVE) are increasingly being explored. According to Snowdon et al. $(1998 ; 2001)$ such a system should be able to support:

Shared Context - Representations of the design problem, which are supposed to enable shared understanding and interactive activity for the group and its different participants. The shared artifacts should be understandable, visible and available for communication and negotiation in the group and should support gesture or pointing during discussions. 
Awareness of others - Through understanding of others tacit knowledge and activities related to the design problem, the team can build up shared understanding that emerges as different stakeholders begin to better understand each other's perspectives.

Transitions between shared and individual activities - relate to the process whereby individual work evolves into collaborative work. It is important that collaborators know what is currently being done and what has been done in the context of the task goals.

Negotiation and communication -Conversations and gestures are crucial for negotiation and communication during collaborative design activities. In this context, face-to-face communication is vital for supporting natural communication through human body features such as facial expression, gestures, postures.

Flexible and multiple viewpoints -Design tasks often require the use of multiple representations and visualisations, each tailored to different points of view and different subtasks and users.

Furthermore, Fischers et. al. (2000; 2005) highlighted two different spaces in their collaborative design environment - action space and reflection space. Action space provides a foundation for creative collaboration between the participants whilst reflection space supports the group members to validate and form their own opinions on the design. They also mention social creative aspect of the design process and stress the importance of interactive collaboration enabling actors to actively contribute rather than having passive consumer roles. More specifically, Fischer et al. (2005) argue that in order to support creation and progression in a social creative design process, it is important to provide systems that can:

- Create awareness of each other's work and provide mechanisms to help draw out the tacit knowledge and perspectives;

- Enable co-creation (in multiple forms: simultaneous, parallel, and serial);

- Allow participants to build on the work of others;

- Provide individual reflection and exploration (e.g. reflection space and action space).

Various types of technology can be deployed to achieve these aims. Multi-touch table interactive systems have been shown to aid such a creative collaborative design process (Arias et al. 2000; Fisher et al. 2005; Voigt, A., Achleitner, E., Linzer, H., Schmidinger, E. and Walchhofer 2003). The multi-touch table has the possibility to give the participant the feeling of an active and meaningful role during the meeting (Jutraz and Zupancic 2012). Still, a common problem with 2D-based design environments is that the information is not presented in such a way that people can easily understand it spatially. In this context, real-time 3D visualizations (e.g. nonimmersive VR) have been shown to offer an efficient communication platform (Balali et al. 2020; Biederman 
1990; Bouchlaghem et al. 2005; Du et al. 2018a; Liu et al. 2020; Mastrolembo Ventura et al. 2019;

Mastrolembo Ventura and Castronovo 2018; Sunesson et al. 2008; Westerdahl et al. 2006). Usability studies of non-immersive VR applications (Roupé 2013; Sunesson et al. 2008; Westerdahl et al. 2006) have shown that VR helps stakeholders with different backgrounds and knowledge to coordinate their perception and understanding of the project. One conclusion has been that VR fosters a more dynamic decision-making process (Roupé 2013). VR has shown the potential to be an effective communication tool that will allow different stakeholders in the planning and design process to better understand the project and each other. VR could provide the stakeholders with opportunities to identify and analyse problems and to jointly coordinate the project with the aim of improving their decision-making and thereby the designed environment. One way in which VR models facilitate decision making is by providing stakeholders with the same frame of reference with respect to the new building and the future environment (Balali et al. 2020; Roupé 2013; Roupé et al. 2014).

With the ability to navigate freely through 3D scenes from a first-person perspective, it is possible to present and communicate ideas regarding future buildings in a way that facilitates understanding among all involved parties, despite their background or professional expertise.

To further enhance the user experience, it is commonly advocated to take advantage of immersive display technologies. Immersive-VR have been shown to be useful per se, whilst stereoscopy, large screen, resolution of the display and wide field-of-view provide additional benefits for perception of space (Castronovo et al. 2013; Heydarian et al. 2015; Paes et al. 2017; Shiratuddin et al. 2004; Thompson et al. 2004). Complementarily, the content of the virtual environment such as textures, known objects etc. are important during spatial processing (Paes et al. 2017). The visual cognitive process includes object recognition processes for sorting the information into patterns, which are then combined and associated with objects that the user has experienced earlier in life (Biederman 1990). These associated objects are then used in the spatial reasoning process of the 3D space. During this reasoning process the mind tries to create an understanding of the visual space within two parallel systems, i.e. a self-centred egocentric reference frame and an environment-centred allocentric reference frame (Plank et al. 2010). Both systems interact during this processing and retrieval of spatial knowledge (Plank et al. 2010). In the egocentric reference frame, the viewer compares him/herself with the objects in 3D space and in the allocentric reference frame the viewer compares relations between object-object or environment-object. Studies have shown that different media and representations facilitate different reasoning processes about the design (Coburn 2017). For instance, flat 2D plan drawings or bird-eye views have been agued to give opportunity for pattern recognition, which is suitable when studying spatial organization, relationship between spaces and objects and orientation of 
different objects i.e. allocentric reference (Coburn 2017). Furthermore, Immersive-VR gives the user the opportunity to compare themselves and their bodies with the environment in a view-dependent process i.e. egocentric reference. Research has also suggested that using the physical-human rotation and movement Immersive-VR provides a better understanding and spatial perception (Paes et al. 2017; Riecke et al. 2010; Roupé et al. 2014; Ruddle and Lessels 2009). In recent years, new Head Mounted Displays (HMDs) have been released, which support better stereoscopy, higher resolution of the display, wider field-of-view, physical-human rotation and movement. Recent studies have shown that space perception in the HTC-Vive starts to be comparable to real world space and distance perception, but still virtual environment feels compressed (Buck et al. 2018; Kelly et al. 2017; Paes et al. 2017). Furthermore, other studies have shown that immersive VR may give a level of understanding and perception of space which is hard to experience using other type of visualizations (Balali et al. 2020; Coburn 2017; Germani et al. 2012; Roupé et al. 2016). Nevertheless, when considering the integration and use of immersive VR within the actual design process, the current adaptation still suffers from a number of limitations, such as navigation and user interface, face-to-face communication and gestures, shared view and space, and multi-view projection (Beck et al. 2013; Kulik et al. 2011; Moghimi et al. 2016; Roth et al. 2016; Roupé et al. 2016).

To date, several different collaborative design systems have been proposed (Faliu et al. 2019; Whyte and Nikolic 2018; Xue et al. 2012). However, when comparing these systems against the recommendations stated by Arias et al. $(2000 ; 2005)$ and Snowdon et. al. $(1998 ; 2001)$, several limitations can be noted. For instance, faceto-face collaboration is difficult in current projection-based and HMD virtual reality systems because they do not provide a visually consistent shared space for all users and have limited support for gesturing, pointing, facial expression, postures (Beck et al. 2013; Du et al. 2018b; a; Gugenheimer et al. 2017; Ibayashi et al. 2015; Kulik et al. 2011; Xue et al. 2012) which would enable the Negotiation and communication and Shared Context aims of collaborative design systems Snowdon et. al. $(1998 ; 2001)$. Other multi-touch table systems enable limited perception of space, provided by a VR system (Arias et al. 2000; Chi et al. 2013; Faliu et al. 2019; Fisher et al. 2005; Xue et al. 2012) and multiple views e.g. Flexible and multiple viewpoints and Shared context. Other systems enable limited creative interactive collaboration with the design (Beck et al. 2013; Du et al. 2018b; Voigt et al. 2009). In a recent paper Faliu et al. (2019) presented a prototype with some similar technological setting as our system, e.g. combining multi-touch table and VR. However, the study targeted the urban planning process and developing a new kind of participatory platform, that enables urban designers, architects, and developers to co-design and communicate their projects with the public. Although there are some 
similarities in the technology, urban planning and urban space is a different context and setting compared to our context of designing buildings e.g. new healthcare environments. More generally, existing work on the use of VR is mostly limited to testing the use of these technologies in experimental situations (Du et al. 2018a; Xue et al. 2012). Our study contributes to this literature both by presenting a new collaborative design system (with seamlessly connected multi-touch table and several VR-systems for interactive and collaborative design) and by evaluating the use of this technology in a real-life design context, with a view on how these new collaborative design systems-based workshops may facilitate the design management process.

In order to address the complexity of design projects such as hospital design, a promising approach would be to combine the CSCW approaches (e.g. multi-touch table) with VR (e.g. Collaborative Virtual Environment (CVE)) and BIM. The collaborative system presented in this paper supports the aims stated by Snowdon et al. (1998; 2001) -Shared Context; Awareness of others; Negotiation and communication; Transitions between shared and individual activities; Flexible and multiple viewpoints. The collaborative design system has been implemented with the intention to support better creative and shared design processes for the involved stakeholders compared to the traditional process and existing systems. To support this process, we have recognized that the system has to support design understanding, participation, communication and collaboration between different stakeholders and that the system must be user friendly. The hypothesis was also that the multitouch table and VR-system complement each other by facilitating both the aims mentioned above (cf. Snowdon et al. $(1998 ; 2001))$ and different design spaces -collaborative (i.e. action space) as well as individual (i.e. reflection space), as mentioned by Arias et al. (2000). Combining technologies to support both self-centred egocentric and environment-centred allocentric reference frames could speed up the understanding, dynamics and transitions during collaborative work through the use of the system (Coburn 2017; Fischer et al. 2005). Building on this hypothesis, our system supports interactive collaboration in both spaces i.e. changes done in the multi-touch table or in VR are updated instantly in both spaces, as illustrated in Fig. 1, 2 and Fig. 3. Our system also supports direct import and interactive use of BIM from the design process. In this particular study, the equipment and furniture used in the system are BIM objects from the Swedish national healthcare database. 


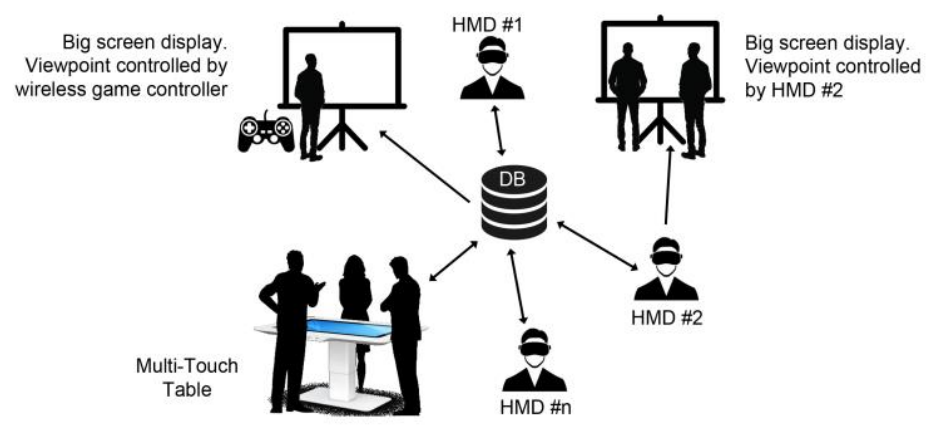

Fig. 1. The collaborative design system supports seamless integration of multiple viewer and interaction clients through a database/server.

\section{The system}

The collaborative design system, Virtual Collaborative Design Environment (ViCoDE), has been developed in the Unity Game Engine using C\#. In essence, it consists of several viewer and interaction clients connected to a central server which manages a database and changes to the system, as illustrated in Fig. 1. The system connects network server and clients, where an IP network with multicast support was realized on top of the network infrastructure. Through the network, any client can interact online with other remote clients. On one of the computers in the network a Redis database is running on a Node.js server. The database in the central server is configured and populated with 3D-components and the designed layout. The initial layout of the proposed design can be imported from the architect's design BIM-software (e.g. Autodesk Revit) and the components in the database are also BIM-objects. The 3D-components in the database have unique IDs (GUID), which makes it possible to traces changes in the variously connected client/viewer applications. Changes of a component's position or rotation in one client are uploaded to the server and then propagated to the other clients. However, as described below, not all clients support all degrees of interaction. The different viewer and interaction clients have different setup and configurations, which facilitate its specific purpose, such as support for the multi-touch table, HMDs (HTC-vive) and VR-viewer for big screen display.

\subsection{Multi-touch table and big screen display}

The layout of the multi-touch table is shown in Fig. 2. It represents the top view of the operating theatre and supports the typical multi-touch pan and zoom features used in most Smart-phones. To better illustrate the scale, a 1x1 meter grid is applied on the floor as a texture. Furniture, medical equipment, walls and static avatars can 
be added, deleted, translated and rotated. All available components are accessible from a scrollable panel on the left side, and are added to the scene using drag-and-drop. The components are BIM-objects coming from the national healthcare database, PTS (Program for Technical Standard). Pressing and dragging a component will translate it whereas ticking it will show a circle for rotation and deleting. Furthermore, components that are mounted in the ceiling are given a different nuance in order to better emphasize their vertical position, see Fig. 2. The interface also supports simulation of how the ceiling pendant systems and its multi-movement arms can be moved around during surgery, making it possible to detect collisions with other equipment.

As seen in Fig. 2 and 3, the contents of the scene can also be displayed on a big screen from a perspective controlled by a wireless game controller, i.e. non-immersive VR.

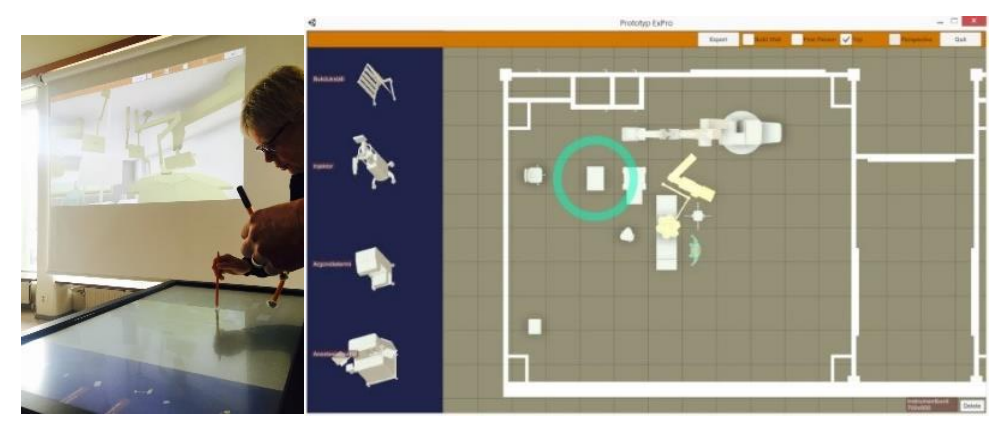

Fig. 2. Left: The multi-touch table used together with a non-immersive VR display. Right: The layout of the multi-touch table screen. Available components are accessible from a scrollable panel on the left side and are added to the scene using dragand-drop

\subsection{VR-system}

The HTC-Vive was used as the immersive display system and a teleportation locomotion mode was used for navigation (Christou and Aristidou 2017). Within the VR environment, components can be translated and rotated. The user of the HMD could interact within the VR environment by picking components and translated and rotated them using the HTC-Vive controllers.

A component will be highlighted if it intersects with any of the HTC-vive controllers and pressing/releasing the trigger allows a user to pick it up and re-place it within the scene. To help the user with the positioning, a component is always restored to its up-right position at the correct elevation above the floor upon release (i.e. to avoid tilting). As illustrated in Fig. 1, 2 and 3, it is also possible to display a user's view from VR on a big screen display. 


\section{The study: use of ViCoDE during collaborative design workshops}

The results of this paper are grounded in two workshops conducted in the spring of 2016 and 2017 , respectively. During these workshops, the collaborative design system was used in real-life contexts of designing operating theatres. The participants were different stakeholders and specialists from healthcare and construction, e.g. theatre nurses, anesthesiologists, architects and project managers, for the intended operating theatres in Skaraborg and Östra Hospital, both located in Västra Götaland, Sweden. All of the participants had previous experience of dialogue-based workshops using traditional information media, such as 2D-drawings and pictures.

From a technical point of view, the main difference between the two workshops was that the first one required an export of the scene to another application to be able to view it in HMD. Also, only one HMD was available and it was not possible to move any of the components in the VR environment. The second workshop, however, used the final system as described in the previous section.
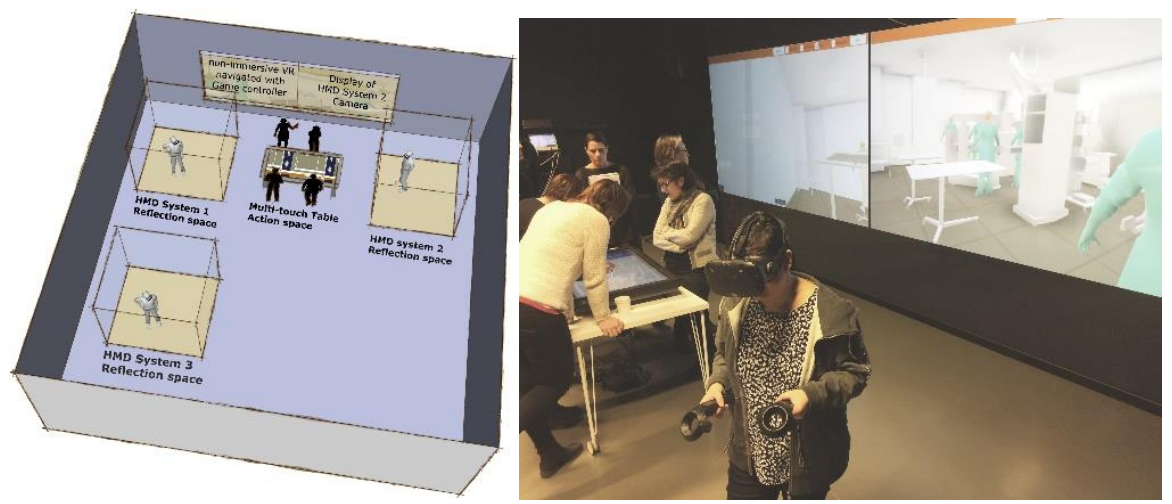

Fig. 3. During workshop 2, three HMD-system were seamlessly used together with the multi-touch table and non-immersive VR. This system supported both collaborative (e.g. action space), as well as individual (e.g. reflection space).

The first workshop had 8 participants and lasted for six hours. It consisted of two different design tasks: 1) designing an operating theatre in a pre-defined space (approx. 8 x 9 meters) and 2) designing an operating theatre without any constraints on room size. The time used for each design task was equal.

The second workshop had 9 participants and lasted for four hours. Fig. 3 shows the room layout and the different collaboration and visualization systems used during the second workshop. The workshop consisted of two different design tasks; the first one being to design an operating theatre in a standard sized room (approx. 8 x 9 meters, approx. 63 square meter), and the second one to design an operating theatre in a room of extended size 
(approx. $10 \times 9$ meters, approx. 82 square meter). The small room located between the two operating theatres is a preparation room, which supports both is operating theatre.

The time used for each of the design tasks was equal. The layout of the proposed operating theatre design came from the architect's BIM project, see Fig. 4.
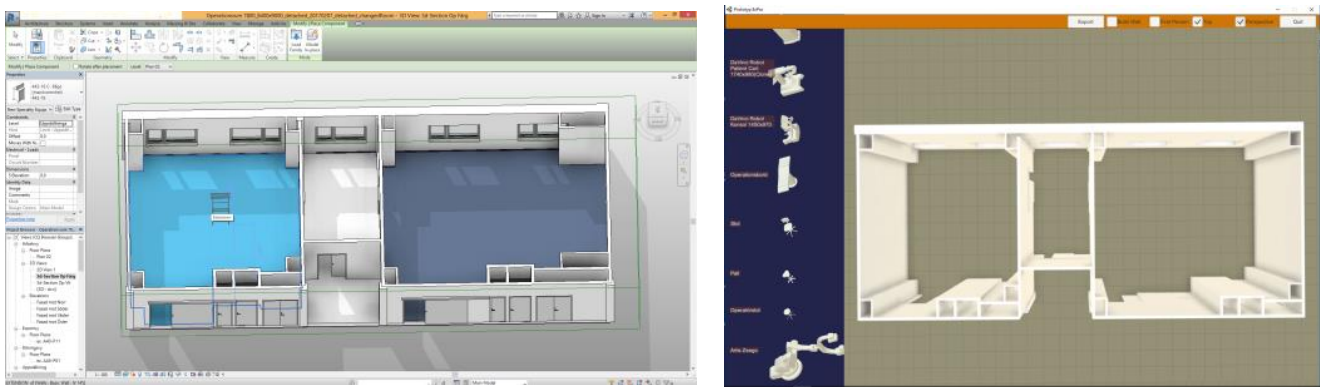

Fig. 4. The left image: the BIM layout of the proposed operating theatre from the project. The right image: the proposed operating theatre layout in. ViCoDE multi-touch table interface.

The workshop started with no equipment and furniture in the operating theatre and the task was to design the layout of the equipment and furniture and to validate if the size was enough. As illustrated in Fig. 5, the workshop resulted in adding furniture and equipment and reducing the size of some rooms (i.e. by adding and moving the walls closer to the equipment).

The result and final version of the large room was approx. $8.9 \times 9$ meters and approx. 71 square meters with a narrower preparation room to fit the general and overall design of the building.
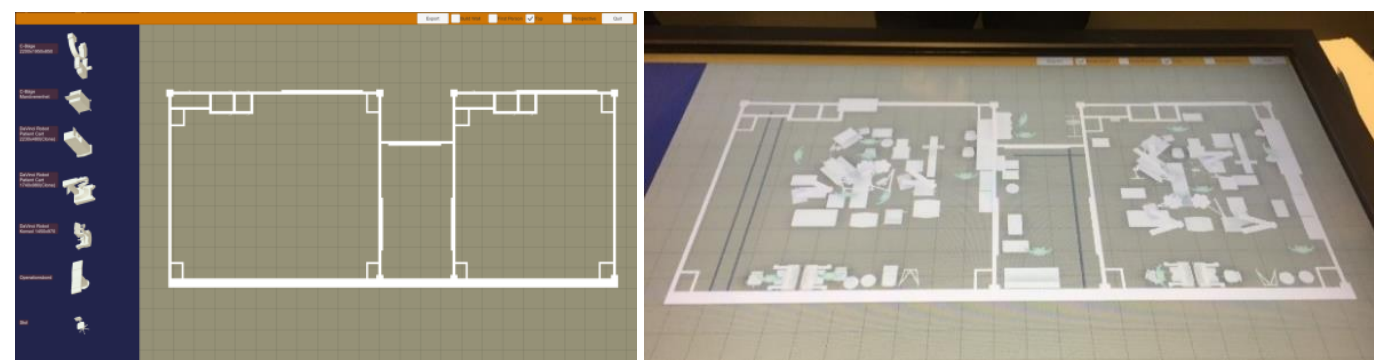

Fig. 5. Left image: The initial BIM layout of walls for the proposed operating theatre design from the architect. Right image: the final result and design from the workshop 2, where new walls (e.g. blue walls) and equipment and furniture are added and the preparation room has also been reduced.

On both occasions, the workshop started with a 10 min introduction of the system to enable the participants to familiarize themselves with the user-interfaces and the overall functionality. 
The study focusses on assessing the use of the virtual collaborative design system (ViCoDE) to support performing design in real-life contexts. The research approach have been inspired by design science research methodology (Hevner 2007), where observations and literature are analysed to develop the artefacts' requirements, which are used as a base for designing and developing the prototype, that are later validated during use in collaborative design workshops, see Fig. 6.

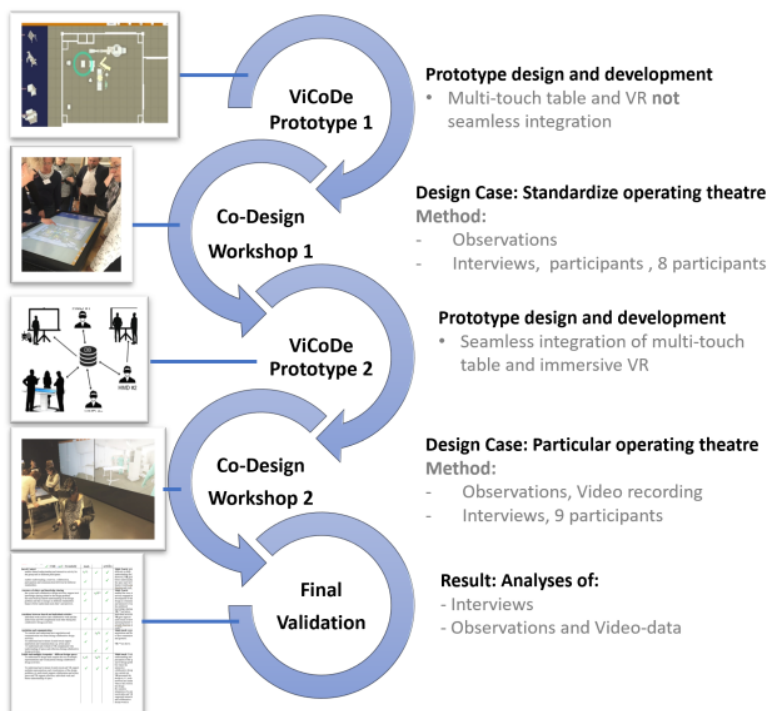

298 Fig. 6. A simplified graphic illustrating of design science research process of this study.

The method during the workshops have taken a qualitative research approach, the study draws on ethnographic and video-based studies of naturally occurring interaction (Heath et al. 2010). During both workshops, qualitative data was collected by means of direct and documented observations as well as informal, semistructured interviews with all the participants about their thoughts and experiences in relation to the collaborative design system. On both occasions three researchers were present. The workshops were facilitated mainly by the architect, with technical support from the researchers.

During and immediately after the workshops, the researchers interviewed the participants regarding their experience and reflection on the collaborative design workshop and the system. The interview data consists of 17 interviews of 5-15 minutes each, which were recorded and transcribed. The main focus of the interviews was to access the participants' views around: 1) how/if the workshop and system supported understanding, participation, communication, and collaboration between the different stakeholders and 2) if the system was user friendly.

The second workshop was recorded with two stationary video cameras which were placed in elevated positions to capture an overview of the participants' movement around and across the different stations in the 
workshop room. The collected corpus of video data consists of 3.45 hours of video data (from the second workshop) which was transcribed for further analysis and later compared to the field notes and interview data in order to reinforce the observations made. The video data was analyzed in a qualitative manner (Heath et al. 2010), by drawing attention to the detail of the natural occurring interactions with the various technologies available in the setting and between the participants as they developed the design of the operating theatre. The verbal interaction between the participants was transcribed by one of the researchers. The transcription was added as subtext in the video data. Subsequently, the researchers separately analyzed and observed the collaboration, conversations and behaviour of the participants during the workshop. After these preliminary analyses, collaborative data review sessions were conducted for further scrutinizing the preliminary observations and developing the thematic categories. The preliminary coding schema and focus during video analyses was based on the literature study connected to collaborative design system (Snowdon et al. (1998; 2001), Arias et al. 2000; Fisher et al. 2005). Additionally, together with interviews and observations during workshop the preliminary categories where recognized. The observed preliminary categories and themes were aggregated and compared across the research team and finally clustered. The result was compared to the field notes and the interviews taken during the workshop. The results that overlapped were selected for further detailed analysis towards the primary codes in table 1.

Table 1. Preliminary coding schema for observations based on CVE-literature.

Drawing on principles of video-based studies methods (Heath et al. 2010), the analysis draws on a collection of illustrative sequences supporting the thematic categories around the use of the collaborative design system during the design workshop. Due to the limited space in this paper, a fragment of approx. 21 minutes of video data was selected from the broader corpus of 3.45 hours of video data to illustrate the collaboration, the verbal and nonverbal interactions and the different behaviour of the participants during the workshop. The fragment selected for detailed analysis presented in this paper was extracted from the final part of the second workshop. The results section begins by summarizing this ( 21 minutes long) fragment, to provide a sense of the interactions emerging in the workshop and to convey the story line. The subsequent subsections of the results present the thematic categories emerging from the data, with a focus on the use of the collaborative design system for developing the design of the operating theatre. 


\section{$4 \quad$ Results}

As explained above, the results section draws on a representative fragment of 21 minutes from the video data. The fragment was extracted from the last part of the second design workshop which lasted four hours. The fragment was selected to illustrate patterns of interaction around the use of the collaborative design system emerging through both workshops. This section begins with a short summary of the workshop to provide context for the selected episode. As the primary codes in table 1, in some cases interact and overlaps, we have choose to present the result use main thematic categories emerging from the data around: Designing using multi-touch table and VR; Different design spaces and understanding of space in different media; Support for better understanding, creativity, collaboration and participation. The section is therefore organized around these thematic categories.

\subsection{Summary and sequence, workshop 2: Designing using multi-touch table and VR}

The fragment presented in this section is part of the second design workshop which consisted of two different design tasks around designing an operating theatre in: 1) a standard sized room (approx. 8 x 9 meters, approx. 63 square meters) and 2) in a room of extended size (approx. $10 \times 9$ meters, approx. 82 square meters). The participants started with the standard sized room, which was the room size decided by the project manager and the project designers as part of the general and overall design of the building. In the following table 2, a summary of observation from first part of workshop 2 is presented connected to coding schema based on CVE-literature from table 1 .

Table 2. Summary of observation from first part of workshop 2, connected to CVE-literature categories.

The final version of the large room was approx. $8.9 \times 9$ meters and approx. 71 square meters compared to the original one which was approx. $10 \times 9$ meters, approx. 82 square meters.

\subsection{Detail description of Observation Case 3: Re-design of the preparation room}

The architect stressed that this new room did not fit the overall design of the building and would probably not be a possible solution. In response, one of the Operations Nurse at the multi-touch table said: - The preparation room feels very big. Can we make the preparation room smaller? (see Fig. 5, the preparation room is the small room which is located between the two operating theatres). Consequently, the architect questioned: - Can we make the 
room narrower? Inquiring the size of the room, the architect added and moved some furniture using the multitouch table whilst communicating verbally with the nurses: - The room is $3.45 \mathrm{~m}$ in width. - I'm trying to understand how big the room is? -What can you compare $3.45 \mathrm{~m}$ with? Any one which have any know references? One of the Operations Nurse at the multi-touch table then said: -How does our current room look like today? Subsequently, two Operations Nurses started to discuss the preparation room size and began to simulate the space by stepping out the room size of $3.45 \mathrm{~m}$ in the background. Joining them, the architect considered that- There is quite a lot of space you can say ... - How big does the room need to be?
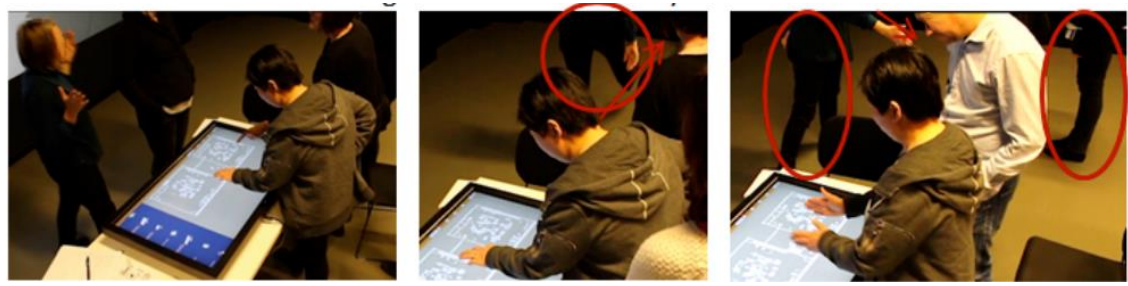

Fig. 7. Image/film sequence from the workshop showing how the architect and Operations Nurses 1 and 2, together tried to create a mental image of how large the room is by stepped-out room in the background as the Anesthetic Nurse adding equipment and furniture's to the preparation room using the multi-touch table.

The architect and Operations Nurses 1 and 2 tried to create a mental image of how the room looks in their current hospital by describing the activities performed and the furniture in the preparation room whilst comparing it with the stepped-out room. In the same time, the Anesthetic Nurse and the other nurses used the multi-touch table to add equipment and furniture to the preparation room, as well as new wall, changing the width of the preparation room to approx. $2 \mathrm{~m}$. Anesthetic Nurse said to the architect and Operations Nurses 1 and 2 - Now you have to go in and test your new preparation room in VR. Checking the multi-touch table, the architect noticed - Now it's only $2.5 m$ wide!!! - Great...

While the Operation Nurse 2 started using the VR-HMD-system, the other participants began to add static avatars / people to understand the size of the new room. Operations Nurse 1 started to refurbish the preparation room, sharing her knowledge on how the room works in her current hospital and expressing her vision for improving it: - There we are two of us ... - I would like to refurbish a little here. -I would like to have preparation there... Trash there, two desk tables for preparation there ... etc. - So I'm standing there and "Operations Nurse 2" there. After Operations Nurse 1 finished her changes of preparation room, she asked Operations Nurse 2 which was in VR - How does it feel now? 
Nurse - Should we move the wall a little? Operations Nurse 2 in VR - Yes. Anesthetic Nurse moved the wall to approx. $3 \mathrm{~m}$ wide while Operations Nurse 2 using the VR validated the room by saying: - It will not feel like you get into a small scrub/room. It's a spacious room - I could stand there the whole day... that's the same room size as my room is today ... Architect says, - Great...

The different participants discussed, tested and validated the new smaller preparation room in the VR-HMDsystem, reaching consensus that the new room worked and the design was satisfactory. Consequently, the architect noted: - If we shrink the preparation room as we have designed here, we can probably managed to fit two of the new version of the operating theaters to the overall design of the building. This was indeed the case, as the final design contained the two resized operating theaters with the newly designed, smaller preparation room between them.

\subsection{User Observation: Different design spaces and understanding of space in different media}

As seen above and from other parts from the video analyses, the data shows that it was difficult to understand the 2D representation of the room (i.e. on the multi-touch table). One example of this challenge was the sequence when the architect and the nurses discussed the size of the preparation room, see Fig. 7. The participants had difficulty to understand the spatial dimensions of the current version of the preparation room. They tried to add known reference object (such as equipment and furniture) into the room using the multi-touch table, but still they thought it was challenging to develop spatial-reasoning with this limited information. They consequently started to physically walk in the background and step out the room size to establish a shared mental image and understanding off the room. This results show that it is difficult to understand 2D layouts (e.g. multi-touch table) and establish shared understanding between different participants with different education, experience and knowledge background. In this setting when only the multi-touch table was used, the participants experienced difficulty in understanding the 2D representation and the design problem fully. Furthermore, they had a limited Shared Context (Snowdon et al. 2001), i.e. shared understanding of the virtual room and the design problem. In this case, the nurses tried to share their knowledge and experience with the architect, by explaining how the existing preparation room looks and works. However, while the architect and the two operations nurses tried to establish shared understanding of the room, the other anesthetic nurse re-designed the room by using the multitouch table and recognized VR as a tool for better understanding of the space and room size. This insight and knowledge came probably from her own experience when they re-designed the size of the large operating theatre rooms in the earlier part of the workshop by using the multi-touch table and the VR-HMD system to move a wall 

and equipment, see described in 4.1 above, and Fig. 5. Invited by the Anesthetic Nurse to experience the room in VR, the architects and the two operating nurses recognized that VR can be used as a tool for better understanding of space. Subsequently, they started to use both VR and the multi-touch table to create shared understanding of the room and its space by re-designing layouts of equipment and furniture as they had discussed in the background. In this context, the multi-touch table, VR-HMD system, 3D-view from the projector, provided supported a shared understanding and communication about the design problem, see table 2, Shared context and Multiple viewpoints. When it comes to spatial understanding and reasoning it is important to recognize the different visual cues in the VR-model. As mentioned in the introduction, the mind tries to create an understanding of the visual space within two parallel systems, i.e. a self-centred egocentric reference frame and an environment-centred allocentric reference frame (Plank et al. 2010). In this context it could be argued that the multi-touch table supported environment-centred allocentric reference frame, where the participants were able to compare relations between object-object or environment-object.

As described in 4.1, the participants used known reference objects such as equipment, furniture and static avatars, while conducting spatial reasoning about the virtual version of the space. When using the multi-touch table, the participants used the 1x1 meter grid applied on the floor as a reference to scale and size of the room. By counting the grid, the user could translate it into square meters and length of the room and for understanding the size of the room. The equipment and furniture enhanced the participants' understanding for evaluating functional and logistic aspects of the room (e.g. environment-centred allocentric reference frame comparing object to object). However, during the middle of the workshops it was observed and recognized that the "2D-top view" on the multi-touch table was not enough, as it was difficult to interpret and understand the space. As the design progressed, the participants started to observe several design errors in the 3D-view from the projector, see Fig. 3. Although the ceiling-mounted equipment had a different nuance to indicate its vertical placement, it was still difficult to perceive it correctly from the top-view in the multi-touch table. In particular, it was difficult to understand how much vertical space the equipment required. After the participants had identified this, they started working with both the multi-touch table and the 3D-view from the projector as a reference. Therefore, it could be argued that the 3D-view from the projector supported view dependent and egocentric reference frame better, as the viewer could compare him/herself with the objects in 3D space in a better way as compared to the multi-touch table. Furthermore, they started to test how the ceiling pendant system and its multi-movement arms moved around during surgery to avoid collision. During the second workshop, the participants also had the opportunity to do this validation in the VR-HMD system. After validating the smaller version, re- designed operating theatre in VR, 
Operating Nurse2 said to Operating Nurse1: "You must try this, it is an entirely different level off experience off the room, when you see it in VR. You can understand it in an entirely different way by actually being there and standing at the operating table and see all the ceiling pendant systems."

The VR-HMD system was emphasized by the participants as enabler for better understanding of how the operating theater would actually function and work. In this context, the users had the possibility to explore a combination of technologies supporting both self-centred egocentric and environment-centred allocentric reference frame and reasoning, which speeded up the understanding and communication and the collaborative work during the use of the system.

During both workshops, the participants used the HMD for reviewing and validating the design of the operating theaters. They had the opportunity to virtually stand by the operating table and make sure that they could see and reach all the equipment and understand the logistics of how the equipment can be moved during surgery. It could be argued that this was enabled by the VR-HMD system supporting better self-centred egocentric spatial reasoning. The participants used physical-human movement and view dependent movement of their body as a reference and they could use both allocentric and egocentric reference frame during spatial reasoning.

Furthermore, it could be argued that the immersive HMD-VR system facilitated a self-centred reflection space where the user had the opportunity to considerate, reflect, validate and confirm the design related to their future work environment and task performed in the operating theater.

In this context, it could also be seen that the multi-touch table facilitated allocentric reference frame, where the overview of the design was presented and where the interactive collaborative design was carried out. The different visualization and interaction techniques gave different support to the users during the workshop, effectively supporting different design spaces. Primarily, the multi-touch table supported a social creative process between the participants, i.e. action or collaboration space, see Fig. 8.

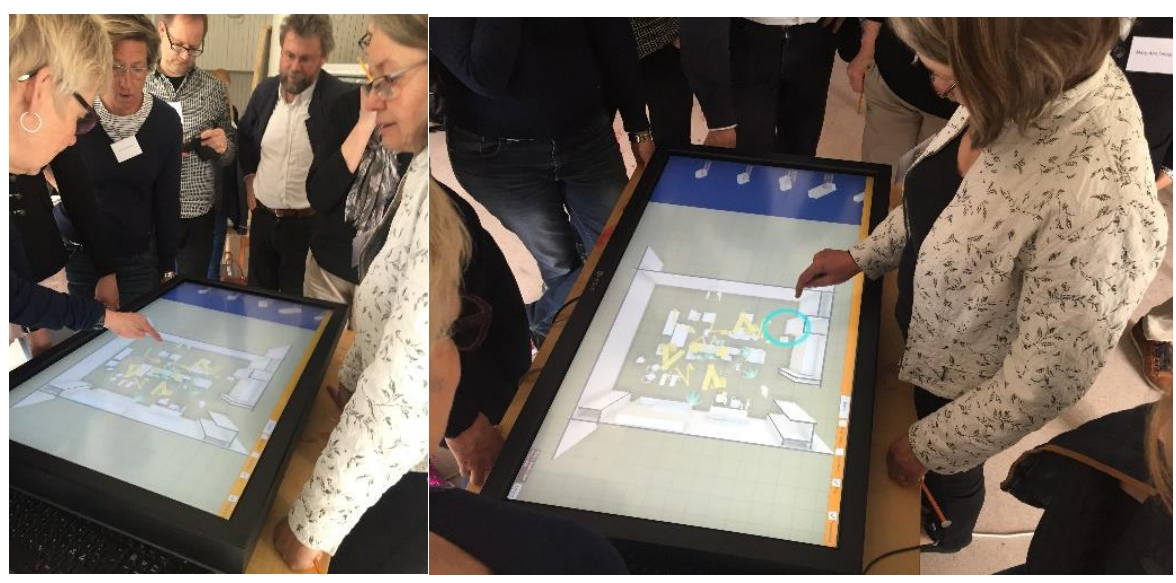


Fig. 8. The multi-touch table supported a social creative process (Picture from workshop 1).

The setting around the multi-touch table was highlighted as the most natural in terms of inter-communication as it supports face-to-face communication and gestures (e.g. (Snowdon et al. 2001)). However, the observations during the workshops also showed that the participants' ability to transform the multi-touch table information into self-made mental images in 3D was demanding, difficult and gave misinterpreted understandings as section 4.1 shows (e.g. limited support for Shared Context and Flexible and multiple viewpoints using multi-touch (Snowdon et al. 2001)). However, the 3D-view from the projector and the VR-HMD system enhanced the spatial understanding. When using the immersive VR-system during the first workshop, the participants wanted to do detailed changes of the design and they communicated the proposed changes to the other participants while they were in the immersive environment. As a result of this, the second workshop had seamless integration of a multitouch table and several immersive VR-systems that supported interactive and collaborative design work in different design spaces - both collaborative (e.g. action space, multi-touch table), as well as individual (e.g. reflection space, VR-HMD). Changes done in one of the spaces were updated in the other design spaces as presented in section 4.1 -e.g. added and moved furniture, equipment, avatars and the wall in the preparation room. Furthermore, the second workshop featured three HMD systems and one of them shared the 3D-view on a projector (see Fig. 3). In this setting it was also possible to make changes to design in the VR-HMD-system. The design changes performed in the VR-HMD-system during workshop 2 were often small distance changes such as moving the furniture and equipment in the egocentric distance near the body. The interviews with the workshop's participants reinforced the idea that the HMD display enhanced the understanding and perception of space and that it enables "more detail changes in the design as displayed at 1:1 scale". These findings support the argument that combinations of technologies support both self-centred egocentric and environment-centred allocentric reference frame, as well as action and reflection space (Arias et al. 2000; Fisher et al. 2005), which speeded up the understanding, dynamics and transitions during collaborative work during the use of the system.

\subsection{User Observation: Support for better understanding, creativity, collaboration and participation}

The observation and video analyses of the workshops indicated that the multi-touch table enabled shared understanding and that resolution and solution of design problems emerged as different participants/stakeholders began to understand each other's perspectives and the design task. The project manager and the architect had the layout and total size of the room (i.e. connected to overall design and construction cost of the building) on their 
agenda, while healthcare staff focused on functionality and task-related aspects of performing surgery. As discussed in section 4.1, the nurses brought their medical practice expertise into the workshop setting and counter-argued the architect's and project manager' attempt to pursue the idea of a smaller operating theatre room. During this process they built a shared understanding and collaboratively recognized the argument for a larger operating theatre room. The architect gained a deeper understanding of the nurses working situation during the workshop while they designed and added furniture and equipment to the small operating theatre room. The video data showed that, during this collaborative design work the architect recognized that a surgery is a lot about logistics around the equipment and patient moves during surgery, where and some equipment is very big and hard to move and rotate such as the $\mathrm{X}$-ray equipment (C-arch). In this context, the design problems resolution and solution lead to the pursuit of another design solution. Whilst traditional design briefing and work involves that the architect takes on the design work to incorporate users' ideas into graphic representations, the presented collaborative design system enabled the possibility to do this together. The technological setting (the multi-touch table) enabled the users to be actively engaged in the development of the design in a dynamic and interactive way as presented, see table 2. For example, during workshop 2, the architect relinquished power over the multi-touch table by stepping back from the table and handing over the pen to the users, as she recognized the transitions of the collaborative workshop into an dynamic co-creation where expertise and knowledge becomes interchangeable, see Fig. 9.
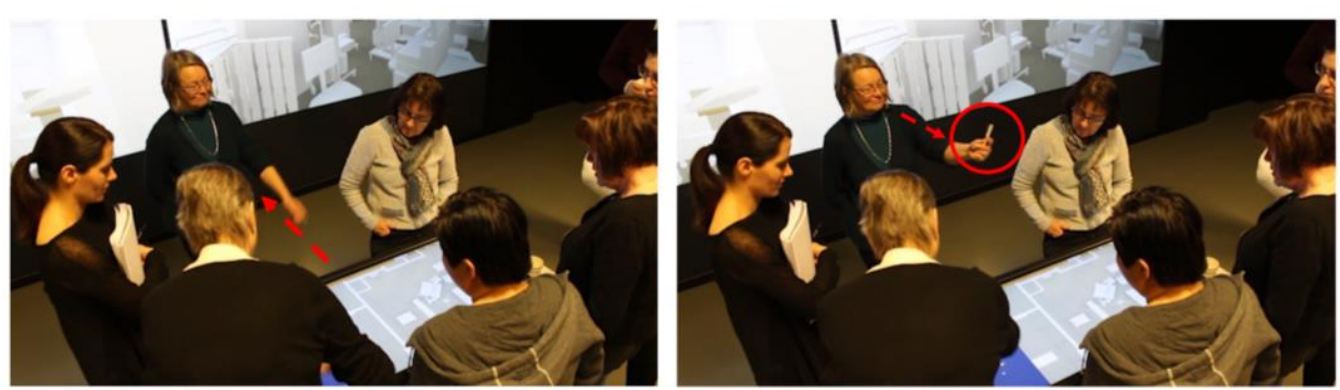

Fig. 9. Imagelfilm sequence (e.g. time frame 14:08-14:09) from the workshop 2 showing how the architect relinquishes power over the multi-touch table by handing over the pen to the users and stepping back from the table as she recognize the transitions of the collaborative workshop into an dynamic co-creation.

This video data indicates a reconfiguration of the design process from the traditional design briefing towards a more collaborative design process, through the architect's giving the other participants the possibility to design through handing over the pen. Consequently, the participants used the drawing and design space via the interactive multi-touch table, starting to collaborate and share knowledge of their medical practice expertise to the design process. During the interviews, the architect highlighted that "the roles were changing, i.e. the 
designers/architects" and thereby more active and involved in the process, as can be recognized from section

4.1. According to the architect and project manager, this had not been the case during earlier, traditional dialogue-based workshops. The architect indicated that the "knowledge and experience transfer from the healthcare staff was better with the collaborative design system". Similarly, the healthcare staff perceived the ViCoDE system as enabler for better understanding of the design and for mobilizing their professional skills during the workshops. It could be argued that these perceived enhancements to the design process were driven by the collaborative design system based workshop's fit with the aims indicated by Snowdon et al. (Snowdon et al. 2001) and Fisher et al. (Fisher et al. 2005), see table 1. During the first workshop, healthcare professionals from different hospitals exchanged knowledge and experience from their particular healthcare environments. The participants mentioned that some professions were missing during the workshop, such as facility management, operations, logistics etc., which they believed would also have benefited from using the system and could have communicated their professional knowledge in the design process.

The architect and project managers argued that this collaborative design system based workshops offered a more efficient process compared to the traditional design briefing. The architect explained that the traditional process often entails long cycles (e.g. weeks) between new proposals and feedback from end-users, whereas this new type of collaborative design process provided almost immediate feedback. The results from workshop 2 (section 4.1) indicate a collaborative and creative process where participants managed to re-design the operation theaters and the preparation room to fit the demands of the overall building design. The resolution of the design problem grows out of the shared understanding that emerges as different stakeholders begin to better understand each other's perspectives (Fisher et al. 2005). In this case the architect recognized that the intended small operation theater was not optimal, but other solutions did not fit the general design of the building: "We have a situation, when we can increase one operation theater and reduce another operation theater. But then we have two rooms that fall out of the overall building design and don't fit." However, the final design result from the workshop addressed this by keeping the two larger version operating theaters whilst reducing the size of the preparation room between them. Complex problems require more knowledge than any single individual possesses (Arias et al. 2000; Fisher et al. 2005), aspect illustrated in the collaborative design workshops data. All involved stakeholders participated, communicated and collaborated with each other to obtain a higher quality outcome. The interviews with the workshops participants indicated the collaborative design system as user-friendly, fun to use, and enabler for better understanding, communication and effective solving of design task. 
The data showed that the participants became easily familiar with the user interface and started to use the system directly after the introduction. The participants indicated that the workshop was a socially creative design process in which all participants could actively contribute with both their knowledge and experience. The data the workshop as a very creative design process, where the different participants shared knowledge and experience. The different visualization and interaction techniques gave different support to the users during the workshop, effectively supporting different design spaces. Primarily, the multi-touch table supported a social creative process between the participants, i.e. action or collaboration space, see Fig. 8 . They used the multi-touch table interface for designing and used the 3D-view from the projector as a visualization medium for understanding 3D-space better and for validation of the design. The workshops indicated that immersive VR (e.g. HMD) gave another level of understanding and perception of space, which was difficult to experience in other type of visualizations. The VR-HMD system also supported ego-centric reflection space where the user could validate the design.

\section{Discussion}

Based on Snowdon et. al. $(1998 ; 2001)$ and Fischers et. al. (2000; 2005), the aim was to design and evaluate the system according to the three criteria of supporting: a creative and shared design processes; better understanding, participation, communication, and collaboration between the different stakeholders; and facilitation of different design spaces - both collaborative and individual (Arias et al. 2000) through the combination of the multi-touch table with the VR-system in a complementary way.

The results based on the use of the ViCoDE system in the collaborative design workshops demonstrate that ViCoDE supported all of the initially stated aims and criteria, see table 1,2 and 3.

Table 3. Summary of validation of the ViCoDe-system and Co-design activities connected the CVE requirements, observation connected to technology and collaborative design activities. 
587 Furthermore, the participants only got approx. 10 min introduction to the system, which proved to be sufficient

588 for the users (e.g. the nurses) to learn how to interact with the system. This shows that the system is user-friendly

589 and there were no barriers for non-technical users to interact and use the system. In fact, it could even be argued

590 that the successful outcome of the workshops was a result of the system actually supporting the above stated collaborative aims. Multi-touch table combined with non-immersive VR, supported interactive collaboration by enabling shared context, understanding and awareness and it provided mechanisms for co-creation and creative collaboration through tacit knowledge transfers, see table 3. By providing seamless integration, usage and collaboration between different systems, ViCoDE facilitated collaboration in simultaneous, parallel, and serial design spaces and support for flexible and multiple viewpoints, transitions between shared and individual activities for different tasks during the workshop, as seen in table 2 and 3. The workshops participants could build on the work of others, which lead to negotiation and communication through collaborative design activities using the multi-touch table. As the results showed, the multi-touch table and the VR-system complement each other by facilitating different design spaces - both collaborative (i.e. action space) and individual (i.e. reflection space) as stated by Fischers et. al. $(2000 ; 2005)$. It could be argued that the immersive HMD-VR system facilitated a selfcentred egocentric reference frame and reflection space, where the user had the opportunity to consider, reflect, validate and confirm the design. In this view dependent self-centred egocentric reflection space, the user/viewer mostly compares him/herself with the objects in designed 3D environment. The multi-touch table facilitated allocentric reference frame where the overview of the design was presented, and the interactive collaborative design was carried out (e.g. action space). In this allocentric reference, the users/viewers studied relationship between spaces and objects and the spatial organization of the designed environment. Action space provides a foundation for creative collaboration between the participants and reflection space provides a foundation for the group members to validate and form their own opinions on the design. Both these spaces connected to the system aim to support Flexible and multiple viewpoints for different subtasks and users and Transitions between shared and individual activities. The bird's-eye view from the multi-touch table gave opportunity for pattern recognition, which is suitable when studying spatial organization, studying relationship between spaces and objects and orientation of different objects e.g. in the allocentric reference frame (Coburn 2017). While the immersive VRsystems enabled the participants to compare themselves and their bodies with the environment in a viewdependent process i.e. egocentric reference frame, which enhanced the spatial understanding of the design environment (Coburn 2017; Roupé et al. 2016). By supporting both media and spaces, the system enabled a better understanding of the designed environment and a better collaborative design process. In comparison to other 
617 systems, our system seamlessly supports interactive collaboration in both spaces, i.e. changes done in both multi-

618 touch table, non- and immersive VR-systems are updated seamlessly in both spaces. However, the results of the

619 workshops showed that the participants primarily wanted to engage in the collaborative design around the multi-

620 touch table and did not take the time to reflect and do design review in the VR-HMD. This might relate to the

621 participants prioritizing the collaborative design activities and changes performed around the multi-touch table. However, when the participants took the time to reflect and perform the design review in VR, they found it useful for contributing to the design. During these design review sessions, the participants stated that the HMD enabled an enhanced level of understanding and perception of the space and of design problems which they could not experience using other media. Our study thus reinforces the argument that multiple design spaces are, indeed, needed in order to foster a collaborative and creative design environment (Arias et al. 2000; Fisher et al. 2005). What was also recognized in this study was that this new type of collaborative design system based process provided almost immediate feedback and change on the design through supporting interactive and dynamic collaborative editing of the design work in comparison to static traditional design reviews. The architect and the project manager explained that the traditional process often entails long cycles (e.g. weeks) between new proposals and feedback from the end-users. As can be seen from the results, workshop 2 was a collaborative and creative process where the participants managed to re-design the operation theaters and the preparation room to fit the demands of the overall building design in less than 4 hours. It could be argued that this type of collaborative design systems and process could achieve a better and more time- and cost-effective design process compared to traditional, dialogue-based design workshops as it encouraged users to solve design tasks more effectively together. In this context, the design management processes are often pressured to reduce lead-time and costs, and to minimize defects and design errors, increase client satisfaction and quality of the new facility (Elf et al. 2015; Lindahl and Ryd 2007). The early stage of the design process is also categorized as a process of gather important end-user feedback regarding the design and its requirements and in this context exist a lot of changes, conflicts, and multiple stakeholders (Arias et al. 2000; Heldal and Roupé 2012). However, as our result show, as all the involved stakeholders participated, communicated, shared tacit knowledge and collaborated with each other, it could be argued that this type of collaborative design system and process may enhance the quality of design compared to traditional design processes. It could therefore be argued that the presented collaborative design systems and process could facilitate and provide a more efficient design management process in the early design stage of a building. The design problems grows out of the shared understanding that emerges as different stakeholders begin to better understand each other's perspectives (Arias et al. 2000; Fisher et al. 2005). 
647 A limitation of the study is that it was conducted on the setting of designing a high-tech healthcare facilities

648 e.g. new robot operation theater. The complex nature of such healthcare environment might have had a positive

649 effect on the results in comparison to less complex facilities such as ordinary healthcare facilities or schools or

650 offices. In this operation theaters context, the operating nurses have the habit to work together standing around

651 the operating table for hours. The similarity of this setting with the multi-touch table might have affected the results of this study. Another limitation is that the study was conducted in a Swedish setting, where the culture is to perform dialogue-based workshops and come to consensus when it comes to group decision-making. How did the Swedish setting influence the outcome of the workshop and the results of this study? These issues would be interesting to be explored in the future using different design settings. For future work it would be interesting to evaluate some of the suggestions given by the participants, such as collision detection among components and color-coding the different equipment according to professional disciplines to better illustrate different responsibilities. Additionally, more usability evaluation of these new emerging technologies and systems would be interesting to explore how they may support new ways of working and how such co-design workshops may improve the design process. Furthermore, seamless integration of BIMsystems would be a natural extension of the system, e.g. trace changes and update the BIM automatically similar to Du et. al. (2018a;b). This would then also make the system support other project types, e.g. schools, urban environments, construction sites. Moreover, the virtual models can function as a platform to engage medical and operational staff in facility co-design. By doing so, digital tools become enablers for evidence-based design outcomes that optimize healthcare facilities to the benefit of patients, staff and visitors alike. This could also facilitate and create a very powerful database and tool for continual development of the facility as well as enabling sharing of concepts and solutions to other projects. If connected to an open database, this could also be part of a larger knowledge-based system, where technologies such as machine learning and recommendation systems can support the users during the workshop. This could eventually be used as a feedback loop to the Swedish County Council standard, Program of Technical standard (PTS), as it would capture knowledge from new design solutions based on different developments of hospitals facilities.

For future work, it would be interesting to evaluate and explore Snowdon et al.' and Fisher et. al.' models (Arias et al. 2000; Churchill and Snowdon 1998; Fisher et al. 2005; Snowdon et al. 2001) in other specific combinations of technologies - such as where users are co-located or distributed-located, using different technologies such as VR-HMD and multi-touch tables and to explore whether there are optimum systems for multi-users interaction. Is the collaborative design around the multi-touch table that important or could collaborative design using VR- 
HMD be enough, or could a distributed-located collaborative design systems support tacit knowledge sharing from experts from different parts of the country/countries or from different hospitals? It would also be interesting to explore how machine learning based recommendation systems could be implemented to support the collaborative design system in a social creative way. Exploring in more depth the effect of other social contexts, such as building on the work of others and the power distribution between users of the system is another interesting avenue for future research.

\section{Conclusions}

The results show that the ViCoDE system and the collaborative design activities, using the multi-touch table and VR-system complement each other very well by facilitating better understanding, participation, communication, knowledge sharing and collaboration among the different stakeholders. For instance, multi-touch table enabled the users to be actively engaged in the development of the design in a dynamic and interactive way and supported knowledge sharing, negotiation and face-to-face communication and gestures, while the users had problem when came to understanding the $2 \mathrm{D}$ and creating spatial-understanding of the design. However, VR gave better understanding of the space and how operating theater would actually function and individual activities in VR gave input to multi-touch users of how the operating theater would actually function and work in reality. By supporting seamless integration of the multi-touch table and VR-system it was recognized that they complement each other very well by facilitating different design spaces - both collaborative, as well as individual. It could be concluded that the ViCoDE supports the aims stated according to Snowdon et. al. ( $1998 ; 2001$ ) and Fischers et. al. (2000; 2005), and that these aims had a positive outcome and enabled co-creation of design in a real-life context.

Furthermore, the real-life design case shows, that collaborative design systems and activities could achieve a better and more time- and cost-effective design process compared to traditional (e.g. 4 hours vs. weeks), as traditional process often entails long cycles between new proposals and feedback from the end-users. The collaborative design systems and activities also encouraged users to solve design tasks more effectively together by communication, knowledge sharing and negotiation in a creative environment, which reduces conflicts of interest. Also, the design and problem grow out of the shared understanding that emerges as different stakeholders begin to better understand each other's perspectives, which could facilitate and provide a more efficient design management process together with end-users. To conclude, the contribution of this study is two folded: presentation of new a collaborative design system and the evaluation of the use of it in a real-life design context, 
which demonstrate how these new technology-based workshops is conducted. By assessing both technical and social factors around the use of this technology in a real-life context, these results contribute to the body of knowledge on how these new types of collaborative design systems and collaborative design workshops could facilitate the design management process.

\section{Data Availability Statement}

Some raw data used in this study were collected during video recording of workshops. This data is sensitive to sharing without the approval from the participants in this study and could be provided with restrictions from the corresponding author by request.

\section{References}

Arias, E., Eden, H., Fischer, G., Gorman, A., and Scharff, E. (2000). "Transcending the individual human mind\&mdash;creating shared understanding through collaborative design." ACM Trans. Comput.-Hum. Interact., 7(1), 84-113.

Balali, V., Zalavadia, A., and Heydarian, A. (2020). "Real-Time Interaction and Cost Estimating within Immersive Virtual Environments.” Journal of Construction Engineering and Management, 146(2), 1-10.

Beck, S., Kunert, A., Kulik, A., and Froehlich, B. (2013). "Immersive Group-to-Group Telepresence." IEEE Transactions on Visualization and Computer Graphics, 19(4), 616-625.

Van Berlo, L. A. H. M., and Natrop, M. (2015). "BIM on the construction site: Providing hidden information on task specific drawings." Journal of Information Technology in Construction, 20(November 2014), 97-106.

Bhatla, A., and Leite, F. (2012). "Integration Framework of BIM With the Last Planner System." 20th International Group for Lean Construction, 10.

Biederman, I. (1990). “Higher-level vision.” Visual cognition and action (vol. 2), 41-72.

Bouchlaghem, D., Shang, H., Whyte, J., and Ganah, A. (2005). "Visualisation in architecture, engineering and construction (AEC)." Automation in Construction, 14(3), 287-295.

Bryde, D., Broquetas, M., and Volm, J. M. (2013). "The project benefits of building information modelling (BIM).” International Journal of Project Management, Elsevier Ltd and APM IPMA, 31(7), 971-980.

Buck, L. E., Young, M. K., and Bodenheimer, B. (2018). “A Comparison of Distance Estimation in HMD-Based Virtual Environments with Different HMD-Based Conditions." ACM Trans. Appl. Percept. Article, 15(15). 
Castronovo, F., Bay, E., Nikolic, D., Liu, Y., and Messner, J. I. (2013). “An evaluation of immersive virtual reality systems for design reviews VIRTUAL REALITY SYSTEMS.” (January).

Chi, H. L., Kang, S. C., and Wang, X. (2013). "Research trends and opportunities of augmented reality applications in architecture, engineering, and construction.” Automation in Construction, Elsevier B.V., 33, 116-122.

Chien, K. F., Wu, Z. H., and Huang, S. C. (2014). "Identifying and assessing critical risk factors for BIM projects: Empirical study.” Automation in Construction, Elsevier B.V., 45, 1-15.

Christou, C. G., and Aristidou, P. (2017). "Steering Versus Teleport Locomotion for Head Mounted Displays." Augmented Reality, Virtual Reality, and Computer Graphics, 431-446.

Churchill, E. F., and Snowdon, D. (1998). "Collaborative virtual environments: an introductory review of issues and systems." Virtual Reality, 3(I 998), 3-15.

Coburn, J. Q. (2017). “An Analysis of Enabling Techniques for Highly- Accessible Low-Cost Virtual Reality Hardware in the Collaborative Engineering Design Process.”

Du, J., Shi, Y., Zou, Z., and Zhao, D. (2018a). “CoVR: Cloud-Based Multiuser Virtual Reality Headset System for Project Communication of Remote Users." Journal of Construction Engineering and Management, 144(2), 1-19.

Du, J., Zou, Z., Shi, Y., and Zhao, D. (2018b). "Zero latency: Real-time synchronization of BIM data in virtual reality for collaborative decision-making.” Automation in Construction, Elsevier, 85(August 2016), 51-64.

Eastman, C. (2016). BIM Handbook BIM Handbook Rafael Sacks.

Elf, M., Frö, P., Lindahl, G., and Wijk, H. (2015). "Shared decision making in designing new healthcare environments-time to begin improving quality.” BMC Health Services Research, 15(1), 1-7.

Faliu, B., Siarheyeva, A., Lou, R., and Merienne, F. (2019). "Design and Prototyping of an Interactive Virtual Environment to Foster Citizen Participation and Creativity in Urban Design." Lecture Notes in Information Systems and Organisation, 55-78.

Fischer, G., Giaccardi, E., Eden, H., Sugimoto, M., and Ye, Y. (2005). "Beyond binary choices: Integrating individual and social creativity.” International Journal of Human-Computer Studies, 63(4), 482-512.

Fisher, G., Giaccardi, E., Eden, H., Sugimoto, M., and Ye, Y. (2005). "Beyond binary choices: integrat- ing individual and social creativity." International Journal of Human-Computer Studies, 12(4), 428-512.

Germani, M., Mengoni, M., and Peruzzini, M. (2012). “An approach to assessing virtual environments for synchronous and remote collaborative design.” Advanced Engineering Informatics, Elsevier Ltd, 26(4), 793-813.

Gugenheimer, J., Stemasov, E., Frommel, J., and Rukzio, E. (2017). “ShareVR: Enabling Co-Located Experiences 

for Virtual Reality between HMD and Non-HMD Users." Proceedings of the 2017 CHI Conference on Human Factors in Computing Systems - CHI '17, 4021-4033.

Heath, C., Hindmarsh, J., and Luff, P. (2010). Video in qualitative research. Sage Publications.

Heldal, I., and Roupé, M. (2012). "Exploring object representations: Virtual reality models for environmental planning projects." Virtual Systems and Multimedia (VSMM), 2012 18th International Conference on, 149156.

Hevner, A. R. (2007). “A Three Cycle View of Design Science Research.” Scandinavian Journal of Information Systems, 19(2), 87-92.

Heydarian, A., Carneiro, J. P., Gerber, D., Becerik-Gerber, B., Hayes, T., and Wood, W. (2015). "Immersive virtual environments versus physical built environments: A benchmarking study for building design and user-built environment explorations.” Automation in Construction, Elsevier B.V., 54, 116-126.

Ibayashi, H., Sugiura, Y., Sakamoto, D., Miyata, N., Tada, M., Okuma, T., Kurata, T., Mochimaru, M., and Igarashi, T. (2015). "Dollhouse VR: A multi-view, multi-user collaborative design workspace with VR technology." SIGGRAPH Asia 2015 Posters, SA 2015, 2-3.

Johansson, M., Roupé, M., and Bosch-Sijtsema, P. (2015). "Real-time visualization of building information models (BIM)." Automation in Construction, Elsevier B.V., 54, 69-82.

Johansson, M., Roupe, M., and Tallgren, M. V. (2014). "From BIM to VR - Integrating Immersive Visualizations in the Current Design Process." Fusion - Proceedings of the 32nd eCAADe Conference - Volume 2 (eCAADe 2014), 195, 261-269.

Jutraz, A., and Zupancic, T. (2012). "Criteria for Cooperative Urban Design through Digital System of Design Tools." 2012 16th International Conference on Information Visualisation, 416-421.

Kelly, J. W., Cherep, L. A., and Siegel, Z. D. (2017). "Perceived Space in the HTC Vive." ACM Transactions on Applied Perception, 15(1), 1-16.

Kulik, A., Kunert, A., Beck, S., Reichel, R., Blach, R., Zink, A., and Froehlich, B. (2011). “C1x6.” Proceedings of the 2011 SIGGRAPH Asia Conference on - SA '11, 30(6), 1.

Lindahl, G., and Ryd, N. (2007). "Clients' goals and the construction project management process." Facilities, 25(3-4), 147-156.

Liu, Y., Castronovo, F., Messner, J., and Leicht, R. (2020). "Evaluating the Impact of Virtual Reality on Design Review Meetings." Journal of Computing in Civil Engineering, 34(1).

Mastrolembo Ventura, S., and Castronovo, F. (2018). "An agenda for implementing semi-immersive virtual reality in design meetings involving clients and end-users." European Conf. on Product and Process Modeling, Copenhagen, 1-9. 
Mastrolembo Ventura, S., Castronovo, F., Nikolić, D., and Ciribini, A. L. C. (2019). "A framework of procedural considerations for implementing virtual reality in design review." Proceedings of the 2019 European Conference for Computing in Construction, 1(July), 442-451.

Moghimi, M., Stone, R., Rotshtein, P., and Cooke, N. (2016). "Influencing Human Affective Responses to Dynamic Virtual Environments.” Presence: Teleoperators \& Virtual Environments, 25(2), 81-107.

Paes, D., Arantes, E., and Irizarry, J. (2017). "Immersive environment for improving the understanding of architectural 3D models: Comparing user spatial perception between immersive and traditional virtual reality systems.” Automation in Construction, Elsevier, 84(August 2016), 292-303.

Plank, M., Müller, H. J., Onton, J., Makeig, S., and Gramann, K. (2010). "Human EEG correlates of spatial navigation within egocentric and allocentric reference frames." International Conference on Spatial Cognition, 191-206.

Riecke, B. E., Bodenheimer, B., McNamara, T. P., Williams, B., Peng, P., and Feuereissen, D. (2010). "Do we need to walk for effective virtual reality navigation? Physical rotations alone may suffice." Lecture Notes in Computer Science (including subseries Lecture Notes in Artificial Intelligence and Lecture Notes in Bioinformatics), 6222 LNAI, 234-247.

Roth, D., Galakhov, D., and Erich, M. (2016). “Avatar Realism and Social Interaction Quality in Virtual Reality.” 277-278.

Roupé, M. (2013). Development and Implementations of Virtual Reality for Decision-making in Urban Planning and Building Design. Doktorsavhandlingar vid Chalmers tekniska högskola. Ny serie.

Roupé, M., Bosch-Sijtsema, P., and Johansson, M. (2014). "Interactive navigation interface for Virtual Reality using the human body." Computers, Environment and Urban Systems, Elsevier Ltd, 43, 42-50.

Roupé, M., Johansson, M., Viklund Tallgren, M., Jörnebrant, F., and Tomsa, P. A. (2016). "Immersive visualization of Building Information Models.” Living Systems and Micro-Utopias: Towards Continuous Designing, Proceedings of the 21st International Conference of the Association for Computer-Aided Architectural Design Research in Asia (CAADRIA 2016), 673.

Ruddle, R. A., and Lessels, S. (2009). “The benefits of using a walking interface to navigate virtual environments.” ACM Transactions on Computer-Human Interaction, 16(1), 1-18.

Shiratuddin, M. F., Thabet, W., and Bowman, D. (2004). "Evaluating the Effectiveness of Virtual Environment Displays for Reviewing Construction 3D Models." Proceedings of CONVR Conference on Construction Applications of Virtual Reality, 87-98.

Snowdon, D., Churchill, E. F., and Munro, A. J. (2001). “Collaborative Virtual Environments: Digital Spaces and Places for CSCW: An Introduction." Collaborative Virtual Environments: Digital Places and Spaces for Interaction, E. F. Churchill, D. N. Snowdon, and A. J. Munro, eds., Springer London, London, 3-17. 
Sunesson, K., Allwood, C., Paulin, D., Heldal, I., Roupe, M., Johansson, M., and Westerdahl, B. (2008). "Virtual Reality As a New Tool in the City Planning Process.” Tsinghua Science \& Technology, 13(October), 255260.

Thompson, W. B., Willemsen, P., Gooch, A. A., Creem-Regehr, S. H., Loomis, J. M., and Beall, A. C. (2004). "Does the quality of the computer graphics matter when judging distances in visually immersive environments?" Presence: Teleoperators \& Virtual Environments, MIT Press, 13(5), 560-571.

Voigt, A., Achleitner, E., Linzer, H., Schmidinger, E. and Walchhofer, H. P. (2003). "Multi-dimensional Digital City Models." The 21th eCAADe - Digital design, 253-257.

Voigt, A., Kieferle, J., and Wössner, U. (2009). "Urban-spatial Experiments with Digital City Models in a Multidimensional VR-Simulation Environment (Urban Experimental Lab)." SIGraDi 2009: Proceedings of the 13th Congress of the Iberoamerican Society of Digital Graphics, 144-146.

Wang, X., and Dunston, P. S. (2008). "User perspectives on mixed reality tabletop visualization for face-to-face collaborative design review." Automation in Construction, 17(4), 399-412.

Westerdahl, B., Suneson, K., Wernemyr, C., Roupé, M., Johansson, M., and Allwood, C. M. (2006). “Users' evaluation of a virtual reality architectural model compared with the experience of the completed building." Automation in Construction, 15(2), 150-165.

Whyte, J., and Nikolic, D. (2018). Virtual reality and the built environment (2nd edition).

Winch, G. M. (2010). Managing construction projects. John Wiley \& Sons.

Xue, X., Shen, Q., Fan, H., Li, H., and Fan, S. (2012). “IT supported collaborative work in A/E/C projects: A tenyear review.” Automation in Construction, Elsevier B.V., 21(1), 1-9. 